\title{
Síndrome de Behcet, revisión de la literatura.
}

Behcet's syndrome, a review of the literature.

Ronny Mera-Flores. ${ }^{1}$, Jorge Cañarte-Alcívar. ${ }^{2}$, Jazmín Anzules Guerra. ${ }^{3}$, Ruth MoreiraVinces. ${ }^{4}$, Rebeka Soasty. ${ }^{5}$, Katiuska Elizabeth Loor Vélez. ${ }^{6}$

Recibido: 18-03-2017 / Revisado: 26-05-2017 Aceptado: 23-06-2018/ Publicado: 01-07-2018

\begin{abstract}
.
DOI: https://doi.org/10.33262/cienciadigital.v2i3.179

Object: To create a source of academic, solid and integral information on Behcet's syndrome. Method: Descriptive documentary study based on contemporary bibliographic sources recovered from Lilacs, Redalyc, ScienceDirect and IntechOpen in English, Portuguese and Spanish. Result: A document was created that reaches the proposed object with a total of 28 articles and texts, physical and digital. Intension: Provide a bibliographic source to Specialists and Students of Health Sciences. Conclusion: The Etiology of Behcet Syndrome starts from a genetic base and an environmental trigger. The most frequent manifestations are mucocutaneous, ocular and vascular with higher incidence, while Neurobehcet, joint and gastrointestinal manifestations do not usually occur frequently.
\end{abstract}

Keywords: Behcet's Disease, Behcet's Syndrome, Triple Symptom Complex.

\footnotetext{
${ }^{1}$ Facultad de Ciencias de la Salud, Universidad Técnica de Manabí; Asociación Científica de Estudiantes de Medicina de la Universidad Técnica de Manabí, Portoviejo - Manabí - Ecuador. rmera1803@utm.edu.ec

${ }^{2}$ Facultad de Ciencias de la Salud, Universidad Técnica de Manabí; Centro Medico Stemmedic - Manabí Ecuador.jcanarte@utm.edu.ec

${ }^{3}$ Facultad de Ciencias de la Salud, Universidad Técnica de Manabí, Portoviejo - Manabí - Ecuador. jzanzules@utm.edu.ec

${ }^{4}$ Facultad de Ciencias Médicas, Universidad Laica Eloy Alfaro de Manabí, Manta - Manabí - Ecuador. ruth.moreira@uleam.edu.ec

${ }^{5}$ Facultad de Ciencias de la Salud, Universidad Técnica de Manabí; Asociación Científica de Estudiantes de Medicina de la Universidad Técnica de Manabí, Portoviejo - Manabí - Ecuador. msoasty9401@utm.edu.ec

${ }^{6}$ Facultad de Ciencias de la Salud, Universidad Técnica de Manabí, Portoviejo - Manabí - Ecuador. kloor3406@utm.edu.ec
} 
Resumen.

Objeto: Construir una fuente de información académicamente proba, solida e integral sobre el Síndrome de Behcet. Método: Estudio descriptivo documental que parte de fuentes bibliográficas contemporáneas recuperadas de Lilacs, Redalyc, ScienceDirect e IntechOpen en inglés, portugués y español. Resultado: Se construyó un documento que alcanza el objeto propuesto con un total de 28 artículos y textos, físicos y digitales. Intensión: Aportar una fuente bibliográfica a Especialistas y Estudiantes de Ciencias de la Salud. Conclusión: La Etiología del Síndrome de Behcet parte de una base genética y un detonante ambiental. Las manifestaciones que se presentan con más frecuencia son mucocutáneas, ocular y vascular con mayor incidencia, mientras que el Neurobehcet, las manifestaciones articulares, gastrointestinales no suelen presentarse con frecuencia.

Palabras claves: Enfermedad de Behcet, Síndrome de Behcet, Complejo de Síntoma Triple.

\section{Introducción.}

El Síndrome de Behcet es una enfermedad de etiología desconocida pero relacionada al genotipo y ambiente del paciente que se caracteriza por afectar a múltiples sistemas, presenta comúnmente aftas orales, úlceras genitales y uveítis, conjunción que ha llevado a que se le denomine Complejo de Síntoma Triple.

El estudio de Behcet como ente patológico constituye una necesidad ineludible para los profesionales de salud y estudiantes, pues del conocimiento, en mayor o menor medida que tengan, se agilitará el correcto cumplimiento de los protocolos de atención integral al paciente.

El impacto del presente trabajo radica en el aporte teórico sobre el Síndrome de Behcet obtenido de fuentes actuales, probas $\mathrm{y}$, en casos relevantes, de obras escritas en un idioma distinto al español, pero cuyo contenido es relevante para el profesional de la salud no anglohablante.

La recopilación de información con las características antes mencionadas fue factible debido a los aportes sobre el Síndrome de Behcet en revistas liberadas y fuentes de producción bibliográfica abierta al público como IntechOpen.

En el desarrollo de la presente revisión se cumplió el objetivo de construir una fuente de información académicamente proba, solida e integral sobre el Síndrome de Behcet orientada a profesionales y estudiantes.

\section{Diseño Metodológico.}


Se realizó una investigación documental y descriptiva. Se efectuó la revisión bibliográfica de documentos recuperados de Lilacs, Redalyc, ScienceDirect e IntechOpen. Se emplearon los Descriptores Enfermedad de Behcet, Síndrome de Behcet y Complejo de Síntoma Triple, y al final se seleccionaron un total de 28 artículos y textos, físicos y digitales, en inglés, portugués y español.

Los criterios de inclusión fueron la disponibilidad del texto completo y tener una antigüedad no mayor a 5 años, los criterios de exclusión fue la inaccesibilidad al texto completo y tener una antigüedad mayor a 5 años.

\section{Desarrollo.}

\section{Behcet.}

El Síndrome de Behcet es una condición inflamatoria crónica multisistémica que induce vasculitis de etiología desconocida, se considera un complejo de síntoma triple por presentar al examen físico aftas orales, úlceras genitales y uveítis. Se caracteriza por compromiso frecuente a nivel mucocutáneo, estomatológico y ocular, puede presentar también manifestaciones neurológicas, cardiovasculares, gastrointestinales, otorrinolaringológicas, ginecológicas y mioarticulares(1-3). Todas las manifestaciones del Síndrome de Behcet presentan vasculitis sistémica, especialmente de venas, misma que es neutrofílica o linfocítica a estudios histopatológicos(4). Investigaciones han descartado la posibilidad de que Behcet sea contagiosa por alguna vía; se presenta exclusivamente en pacientes con predisposición genética cuya expresión debe ser inducida por factores externos; medio ambiente, agentes infecciosos o respuesta inmunitaria.(1,5).

\section{Epidemiología.}

El Síndrome de Behcet mantiene una distribución global, no obstante su prevalencia pondera en Irán, Irak, Uzbekistán, Turkmenistán, China y Turquía; países que forman la antigua ruta comercial de la seda(6,7), hecho atribuido a que sus pobladores presentan una prevalencia alta del Antígeno Leucocitario Humano HLA-B5 y HLA-B51(8,9), sobre este primer antígeno se ha determinado la distribución su prevalencia de la siguiente manera: $30 \%$ en Pobladores del Norte de India, 20\% en Pueblos del Mediterráneo y 36,9\% en Nipones(9). Es significativamente menor su incidencia en Estados Unidos y el Norte de Europa, y en otros Países de América es considerada como una enfermedad rara(7) por las casi nulas cifras reportadas. En estos últimos condicionantes geográficos la existencia de casos tiende a ser relacionada con descendencia de personas que provienen de los países relacionados a la ruta de la seda, lo que ensalza la participación de genes predisponentes a la patología(4). 
La prevalencia del Síndrome de Behcet en Turquía es de 20 a 602 por cada 100.000 habitantes(1,2,4), en Israel va de 50 a 185 por cada 100.000 habitantes, en Irán de 16,7 a 100 por cada 100.000 habitantes, en Iraq 17 por cada 100.000 habitantes, en Japón de 7 a 13,5 por cada 100.000 habitantes, en China 2,62 por cada 100.000 habitantes, en Kuwait 2,1 por cada 100.000 habitantes, en Italia de 4,1 a 15,9 por cada 100.000 habitantes, en Germania 0,9 por cada 100.000 habitantes(1), en países Europeos la prevalencia es de 0,27 a 7,5 por cada 100.000 habitantes, mientras que en U.S.A. se reporta una prevalencia de $0,33(1,2)$.

La incidencia del sexo biológico como factor predisponente a padecer el Síndrome de Behcet tiene un sesgo metodológico debido a la heterogeneidad de tal elemento de la investigación. Los estudios más recientes coinciden en que la relación hombre:mujer varía en función del país estudiado, Emmi y Prisco afirman que en Egipto es mayor la prevalencia en el sexo masculino, con una relación de 5,37:1, mientras que en U.S.A. esta se invierte, con una relación de 0,38:1(3)(3), en tanto Gonul y Kartal explican que en países Asiáticos, como Japón y Korea del Síndrome de Behcet es más frecuente en mujeres, en contraste los países de Oriente Medio presentan predominancia en pacientes masculinos, lo que coincide con la investigación de Ishigatsubo y Castillo $(1,2,5)$, este último agrega que en Alemania, Japón, Brasil y U.S.A. es más común en mujeres. Las investigaciones citadas ibídem en que el desarrollo de la enfermedad es más severo en hombres.

El Síndrome de Behcet inicia en pacientes de 20 a 40 años de edad(1-5,7,8), el curso de la enfermedad es más severo en pacientes menores de 25 años(8) y a pesar de que su diagnóstico es inusual en pacientes pediátricos o mayores de 50 años, se han reportado casos. $(4,8)$.

\section{Genética.}

Se conoce de forma parcial el rol, presumiblemente, clave de la genética en la etiología del Síndrome de Behcet; el cromosoma 6p21, relacionado íntimamente con la respuesta inmune, codifica al Antígeno Leucocitario Humano B5 (HLA-B5), del Complejo Mayor de Histocompatibilidad clase I (MHC), lo que postula a Behcet como una MHC-I-opatía. La presencia del HLA-B5 y el alelo HLA-B5*1, subtipo B*51:01, que tiene mayor grado de polimorfismo en el genoma humano, es un rasgo altamente predisponente relacionado aproximadamente al 40-80\% de los casos en función del grupo étnico. $(1,2,10)$ Sin embargo, no se puede disociar de la aparición de esta condición a otros factores como el medio ambiente, microorganismos e infeccione y nuevas mutaciones recientemente identificadas, que se relacionan con dicotómicamente con el HLA-B5*1(1). Se considera la hipótesis de que HLA-B*51 presenta antígenos propios a los Linfocitos T lo que conduce a una activación de la respuesta inmunitaria(10).

Estudios han permitido determinar que HLA-B*15 y HLA-B*27 implican cierto grado de riesgo mientras HLA-B*49 y HLA-A*03 cumplen una función protectora, HLA-B*57 es un marcador de riesgo para la población española y HLA-A*26 lo es para la población 
japonesa(1), HLA-B*52, idéntico al HLA-B*51 a excepción de dos residuos de aminoácidos no está relacionado con el Síndrome de Behcet(3). En Italia, Portugal Alaska y Canada la positividad para el alelo HLA-B*51 es semejante a la de la zona de la Ruta de la Seda, donde la prevalencia del Síndrome de Behcet esta elevada, en contraste, en estos países la prevalencia es de 2 casos por cada 100 mil personas(2,3), lo que permite inferir la posible existencia de factores intrínsecos detonantes o predisponentes en la Ruta de la Sede.

Los Estudios de Asociación de Genoma Completo, GWAS por sus siglas en ingles han permitido identificar y sumar nuevas variantes genéticas asociadas a Behcet, estos candidatos son los loci KIAA1529, CPVL, LOC100129342, UBASH3B y UBAC2, y otros genes como IL10, IL23R-IL12RB2, STAT4, TNFAIP3, CCR1-CCR3, KLRC4, FUT2, IL12A, IL23R, TLR4, MEFV, NOD2, ERAP1, HLA-A*26, los no relacionados con HLA: ICAM1, VEGF, eNOS, Factor de Coagulación V.

\section{Relación de Infecciones e Inmunidad en Behcet.}

Como se ha establecido ya, existe un componente genético que vuelve susceptibles a los pacientes a padecer el Síndrome de Behcet, sin embargo, no es suficiente justificativo para la etiología de la enfermedad, hecho que da luz a la participación activa de un componente ambiental; donde resaltan los agentes infecciosos, sobre todo bacterias y virus, y la respuesta inmunológica que desatan. Se considera a los patógenos habituales de la orofaringe como principales agentes patogénicos en relación con la frecuencia de las aftas orales, entre los que destacan los estreptococos(1).

Existe una proliferación característica de Streptococcus Sanguinis en la flora oral de los pacientes son Síndrome de Behcet, y en el suero de estos pacientes se identifica muy frecuentemente anticuerpos contra S. Sanguinis y S. pyogenes, relacionándolos con ulceración oral por hipersensibilidad a los antígenos estreptocócicos. A pesar de la relación expuesta, se ha demostrado que un antígeno no proteico común a muchas bacterias, incluidas E. Coli y S. Aureus, activan a las células $\gamma \delta$-T en pacientes con Síndrome de Behcet y que las enterotoxinas estafilocócicas activan a los Linfocitos $\mathrm{T}$ de estos pacientes $(1,3)$.

En cuanto a infecciones virales, los primeros postulados tuvieron su génesis en investigaciones de Hulusi Behcet. Investigaciones permitieron identificar el genoma del HSV-1 en ulceras orales y genitales, leucocitos, sangre, saliva y suero de pacientes con Síndrome de Behcet, en contraste a la concepción de que infecciones por HSV-1 jugaban un papel relevante como tales en la patogénesis, actualmente se acepta más la teoría de que la respuesta inmune ante este virus participa en la patogénesis del Síndrome de Behcet(1). 
No se ha logrado determinar, por falta de estudios o resultados la participación directa, por infección, o indirecta, por la respuesta inmune inducida, de los Virus de la Hepatitis A, B, C, E, G, Parvovirus B19, VIH, VZV, Citomegaloviruas y EBV, pero existen indicios que son actualmente objeto de estudio( 1,3$)$.

Indiferente de cual sea el agente infeccioso que inicie la respuesta inmunitaria, esta ampliamente aceptada la participación de las Proteínas de Choque Térmico a causa de una reacción cruzada. El mimetismo molecular es de igual forma objeto de estudio, sobre todo el antígeno $\mathrm{S}$ de la retina en relación con HLA-B51 y HLA-B27 y el gen Bes-1 del S. Sanguinis con el péptido ganglionar intraocular humano Brm-3b. Este último podría explicar en cierta medida la uveítis(1).

Estudios a través del tiempo han permitido identificar que los niveles bajos de hepcidina salival y sérica, péptido antimicrobiano, se relacionan con el desarrollo de las aftas orales, que el bajo nivel de lectina ligadora de manosa está relacionada a la colonización de S. Aureus en pústulas del paciente con Síndrome de Behcet y que los géneros Roseburia y Subdoligranulum de la microbiota intestinal están disminuidos, junto con los niveles de producción de butirato(1).

En cuanto al papel específico de componentes específicos de la respuesta inmune se ha demostrado una hiperfunción de los Linfocitos Th17, presentes en lesiones cutáneas e inflamatorias a nivel cerebral, probablemente por una condición de hipersensibilidad de estos Th a IL23 e IL12, induciendo la producción de IFN $\gamma$, mismo que se encuentra en altas concentraciones junto con la proteína reguladora de la secreción de IFN $\gamma$, Txk. Las citocinas y quimiocinas de Linfocito Th1 se presentan elevadas en sangre periférica y lesiones de pacientes con Síndrome de Behcet(1,2).

Actualmente se acepta que los Linfocitos Th17, productores de IFN $\gamma$, a postre sufren un cambio de tipo diferenciándose a Linfocitos Th1 no convencionales, este último subtipo de Linfocito T participaría en la fisiopatología del Síndrome de Behcet debido a su mayor afinidad por lesiones inflamatorias que el Th17 inicial. GWAS ha permitido dar luz a la hipótesis de que la GTPasa participaría en la diferenciación de Th17 a Th1 no ordinario en pacientes con susceptibilidad genética para el Síndrome de Behcet(2,3).

Respecto a los Linfocitos T Reguladores, su papel en la fisiopatología y patogenia del Síndrome de Behcet no es clara, sin embargo, estudios en que las concentraciones del gen Foxp3 y Linfocitos Treg $\mathrm{CD}_{2} 5^{+}$se encontraban elevadas en el líquido cefalorraquídeo y que a la citología estos Treg presentaran plasticidad similar a los L Th17 apuntan a la participación de estas células reguladoras en Neurobehcet(2). 
El papel de los Linfocitos B, sobre todo los subtipos Linfocito B Activado CD80 $0^{+}$y

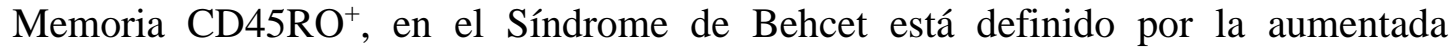
secreción por parte de este grupo celular de inmunoglobulinas de manera espontánea, a pesar de la reducida estimulación por mitógenos. Estudios Clínicos han identificado en úlceras orales y lesiones eritematosas nodulares IgM y la Proteína 3 del Complemento (C3), auto-anticuerpos dirigidos contra células endoteliales e inmunoglobulina sérica elevada en estos pacientes. Se estudia la relación del Virus de Epstein-Barr debido a ensayos de activación en que este indujo una sobreproducción de inmunoglobulinas $(2,3)$.

Se considera que los auto-anticuerpos participan activamente en el desarrollo de la fisiopatología del Síndrome de Behcet debido a una alta tasa de anticuerpos positivos anti- $\alpha$-enolasa, antígeno diana del anticuerpo celular anti-endotelial, Kinectin y cofi lin 1 y mayores títulos de anti-troposimina.

\section{Características Clínicas.}

Las manifestaciones clínicas son diversas, capaces de afectar a diversos tejidos como órganos. Es de interés conocer acerca de lo establecido por Criterios Internacionales para la Enfermedad de Behcet (ICBD), para probar si una persona padece o no la enfermedad, dando positivo si la puntuación es $\geq 4$ puntos, el puntaje que se registra es el siguiente: Dos puntos si presenta Lesiones Oculares, Aftas Oraleso Aftas Genitales. Un punto si presenta lesiones cutáneas, manifestaciones de comprimiso del sistema nervioso central, evidencia de compromiso vascular y la prueba de patergia positiva. Los puntos son individuales para manifestación $(11,12)$.

\section{Reacción de Patergia.}

Característica del síndrome de Behcet, se le define como el estado de hipersensibilidad cutánea identificado por la presencia de pústula estéril, luego de 24 a 48 horas de la punción cutánea con aguja. A pesar de que la causa es desconocida, se la relaciona con la afluencia de células polimorfonucleares (PMN) a las capas más superficiales de la piel, debido a un aumento de la actividad quimiotáctica, fagocítica y de la migración(12).

Se considera positiva la reacción de patergia cuando tras realizar la punción aparece una masa pequeña rojiza en la epidermis, demostrando que el sistema inmunitario reacciona exageradamente frente una lesión menor(1,13).

\section{Implicaciones Mucocutáneas.}


La característica clínica más común entre los pacientes es que presentan ulceras recurrentes múltiples, dolorosas, con fondos blancos en la cavidad oral. La ulceras genitales son las únicas que dejan cicatrices, sin embargo, son menos frecuentes que las anteriores y aparecen tras las yagas recurrentes en forma de ulceras activas o con cicatrices recuperadas. La presencia del eritema nodoso es la manifestación más habitual de las lesiones cutáneas. La aparición de pápulas pustulosas y formes son también comunes. Se puede encontrar en el paciente exantemas, foliculitis, y pseudofoliculitis(9).

\section{Manifestaciones Estomatológicas y Gastrointestinales.}

Entre el 3 y $30 \%$ de los pacientes presentan manifestaciones gastrointestinales. A lo largo de la mucosa del tracto gastrointestinal se evidencian ulceraciones, existen a la par alteraciones y aumento de la pared del íleon y ciego que tienden a orientar un diagnóstico erróneo de enfermedad inflamatoria intestinal. Los signos y síntomas que más comunes suelen ser inflamación intestinal crónica, dolor abdominal, diarrea, sangrado intestinal y/o ulceraciones difusas, profundas, en esófago y/o el recto(14).

\section{Implicaciones Oculares.}

Se presenta en los primeros 5 años de evolución en el 25\% a 75\% de los pacientes. Entre los factores de riesgo se han identificado al género masculino, los brotes oculares repetidos, la presencia de HLA-B51 y el tratamiento poco agresivo o tardío como agentes que predisponen al paciente a manifestar implicación ocular. La panuveítis y vasculitis como la inflamación del segmento posterior, que son de alto potencial destructor contra la integridad del globo ocular y, en consecuencia, la visión, es la características esencial del Síndrome de Behcet a este nivel(14).

El 70\% de los pacientes presenta afección ocular como uveítis anterior aguda con hipopión, vasculitis retiniana a nivel tanto de venas como arterias, neuropatía del nervio óptico, infiltrados retinianos, escleritis y organización vítrea que pueden ocasionar ceguera(14). Los niveles de IL-4, IL-6, IL-10 IL-17A, IL-17F, IL-31, IFN- $\gamma$ y TNF $\alpha$ se encuentran elevados en pacientes con EB que presentan uveítis recurrente(15). Se han definido tres tipos de uveítis en asociación a EB, estas son:

Uveítis Anterior; iridociclitis recurrente con hipopión es una Inflamación no granulomatosa aguda que sucede en la cámara anterior del ojo (iris, cuerpos ciliares, etc.) Los pacientes con EB pueden cursar con dolor periorbital, fotofobia, visión difusa, enrojecimiento, miosis reactiva, lagrimeo y presentar blanqueamiento parcial del área visual provocando ceguera. Estas manifestaciones desaparecen a las pocas semanas. Uveítis Intermedia; El síntoma más característico es miodesopsias aunque en ocasiones inicia con pérdida de visión central por consecuencia del edema macular crónico. 
Mientras que el signo más característico es la vitritis. Uveítis Posterior o Uveorretinitis; vasculitis con sangrado y exudado: la vitritis y la perivasculitis retiniana abarca tanto arterias (periarteritis) como venas (periflebitis). La periflebitis se identifica por una turbidez blanco esponjoso que rodea el endotelio, con fugas irregulares en la angiografía por fluoresceína. La vitritis se determina por la infiltración celular de productos del vítreo junto con la participación del segmento posterior generando opacidad del vítreo que es habitualmente severa con una grave inflamación del segmento posterior.

La retinitis se identifica por infiltrados superficiales difusos de color blanquecino-amarillento solitarios o multifocales, provocando que la retina tome una apariencia turbia con obstrucción de sus vasos. En el segmento posterior se presenta también ataques de vasculitis oclusivas de la retina. La complicación más común de la EB ocular es edema macular quístico(14).

\section{Manifestaciones Neurológicas y Neuropsicológicas: Neurobehcet.}

Las manifestaciones neurológicas son menos frecuentes, al inicio de la enfermedad, solo del 5 al $10 \%$ de los pacientes lo padecen y es una de las complicaciones menos benignas de la afección $(2,11)$.

Su inicio suscita tras afectación primaria por vasculitis en el sistema nervioso, trombosis de los senos venosos, como afección más usual(16).Las complicaciones neurológicas provocadas por manifestaciones sistémicas como accidente cerebrovascular cardioembólico que suelen ser secundarias a cardiopatía por Behcet. Las complicaciones del tratamiento de la enfermedad como la neurotoxicidad inducida por la ciclosporina también puede desatar Neurobehcet(16).

Un NeuroBehcet con más de 15 años de evolución se caracteriza por atrofia de ganglios de la base y formación de quistes en lesiones antiguas. En cuanto a la distribución de las manifestaciones, pueden ser intraparenquimatosas, parenquimatosas y extraparenquimatosas. En la fase aguda de las lesiones intraparenquimatosas sucede infiltración de células mononucleares (monocitos, linfocitos T, y escasos linfocitos B) alrededor de los pequeños vasos produciendo una muerte neuronal. Las lesiones parenquimatosas son focales o multifocales y se clasifican en agudas y crónicas o progresivas; los trastornos piramidales, los signos de lesión cortical y trastornos cognitivos y/o de la conducta son las manifestaciones más usuales, mientras la mielitis y el pseudotumoración cerebral son manifestaciones poco frecuentes y precedidas por su gran severidad(16).

La aparición de lesiones típicas de Neurobehçet pueden generar síntomas psiquiátricos, dando lugar a Neuro-Psico-Behçet (NBP), es decir, presencia manifestaciones psiquiátricas junto con las afecciones neurológicas(17). Estos pacientes suelen iniciar con manifestaciones 
de ansiedad y depresión, junto a cambios del comportamiento como euforia, pérdida del juicio, indiferencia y desórdenes psicomotores.

\section{Manifestaciones Cardiovasculares.}

Afecta al sistema cardiovascular induciendo perivasculitis distintiva que puede involucrar venas y arterias de todos los calibres(2). Se incluyen procesos patológicos como aneurismas y oclusiones por vasculitis arteriales sistémicas y pulmonares, oclusiones venosas y várices, estas condiciones y sus complicaciones tienden a empeorar el proceso sistémico(6).

Las manifestaciones arteriales son a menudo responsables de signos sistémicos: fiebre, adelgazamiento, síndrome inflamatorio biológico. Las manifestaciones más específicas son dolor abdominal o torácico en función de la localización de las lesiones, signos isquémicos tras la lesión, en particular claudicación de los miembros o accidentes isquémicos cerebrales, hemoptisis en caso de afectación arterial pulmonar(4), tromboflebitis superficial, trombosis venosa o arteritis. La arteritis se manifiesta por fenómenos oclusivos-trombóticos y aneurismáticos que afectan de forma discriminativa a la aorta, arteria pulmonar, arteria poplítea, arteria femoral, arteria subclavia, y menos frecuente carótida común; y son responsables de eventos hemorrágicos o infartos en diferentes órganos(5). Las principales manifestaciones son la trombosis venosa y arterial y aneurismas arteriales, también se puede presentar trombosis intracardiaca seguida de pericarditis, miocarditis, enfermedad coronaria y valvular(2).

Los aneurismas y los pseudoaneurismas son las principales manifestaciones a nivel arterial, específicamente en la aórtica y menos común en la periferia. Las lesión subyacente es arteritis necrosante, infiltración inflamatoria intensa por neutrófilos, linfocitos y células plasmáticas con varios histiocitos y eosinófilos que involucran destrucción y pérdida de las fibras elásticas y musculares por motivo de la proliferación y una oclusión de la vasa vasorum que eventualmente llevará a una fibrosis.

La obliteración inflamatoria del vaso puede causar la formación de un pseudoaneurisma o la ruptura de la pared arterial(2). El retraso en el diagnóstico y el inicio del tratamiento de esta complicación puede ser responsable de la rotura del aneurisma con hemoptisis masiva de una elevada mortalidad, tasa que se ve potenciada si la complicación se presenta en adultos mayores, en quienes la anemia es un problema frecuente $(5,18)$.

\section{Manifestaciones Otorrinolaringológicas.}

En pacientes con manifestaciones otorrinolaringológicas, las úlceras orales son las más frecuentes, menos frecuentes son las úlceras orofaríngeas pudiendo manifestarse con odinofagia secundaria que puede conducir a un diagnóstico erróneo de amigdalitis aguda, en 
índices menores, se presentan síntomas audiovestibulares como hipoacusia neurosensorial, síndrome vertiginoso e hipofunción vestibular bilateral(19).

\section{Manifestaciones Musculares y Articulares.}

La artralgia o artritis en forma de mono u oligoartritis que afectan las rodillas, tobillos o muñecas constituyen el dato característico inicial que de más de la mitad de pacientes con síndrome de Behcet. La artritis en esta condición es intermitente y no erosiva por cuanto es fundamental diferenciarla de manifestaciones articulares relacionadas a enfermedad reumática u otras formas de artritis seronegativa $(3,4)$.

El síndrome de MAGIC (mouth and genital ulcers with inflamed cartilage syndrome "úlceras bucales y genitales con síndrome de cartílago inflamado") es una enfermedad que presenta solapamiento entre la policondritis recurrente y la enfermedad de Behcet(3).

\section{Implicaciones Audio Vestibulares.}

En el síndrome de Behcet, las manifestaciones clínicas en el área audiovestibular no son extrañas, los primeros síntomas afectan al sistema nervioso central(19,20). Se han observado cuadros compatibles con neuronitis vestibular, constituyendo una de las primeras afectaciones del sistema nervioso central (SNC) a lo largo de la evolución de la enfermedad. Presencia de de cuadros veriginosos inespecíficos y en otros pacientes desarrollaron hipoacusia neurosensorial para altas frecuencias(19).

\section{Manifestaciones Ginecológicas.}

En la inspección física se pueden destacar úlceras aftosas pequeñas en la mucosa labial. Edemas valvulares asociados a úlceras en lugares como el clítoris, labios mayores y en todas las caras de la vagina con abundante secreción amarillo-verdosa(21). Las ulceraciones tienden a ser más frecuentes y más graves en los ciclos premenstruales y menstruales. Incluso durante la gestación suelen ser menos importantes a pesar de que pueden persistir durante todo el embarazo y agravarse tras el parto(22). Es importante examinar las características de las lesiones en los pacientes para evitar diagnósticos erróneos, como vaginitis o vaginosis(23).

\section{Behcet en Pacientes Pediátricos.}

Investigaciones en pacientes adolescentes con un promedio de edad de 12 años se pudo constatar que las mujeres y personas de raza blanca, eran las más susceptibles al síndrome de 
Behcet. El predominio en antecedentes familiares con síndrome de Behcet es alto. El promedio de diagnóstico de la enfermedad fue un periodo de 4,6 años debutando con úlceras orales y otros síntomas en general, al pasar el tiempo durante la evolución de la enfermedad se presentaron aftas genitales, afectaciones oculares, neurológicas, digestivas y del $\operatorname{SOMA}(24)$.

\section{Biomarcadores y Diagnóstico.}

No hay una prueba serológica única para el diagnóstico. Los estudios repetidos que evalúan la prevalencia de pruebas de laboratorio reumatológicas estándar, como anticuerpos antinucleares, anticuerpos anticitoplasma de neutrófilos (ANCA), etc., no han demostrado una mayor prevalencia de esos marcadores en Síndrome de Behcet. La asociación del alelo del antígeno leucocitario humano HLA-B51 (subtipo B * 51: 01) es bien reconocida como el gen de susceptibilidad genética más fuerte hasta el momento entre los pacientes con Sindrome de Behcet genéticamente predispuestos, las investigaciones posteriores con la última tecnología han llevado a la identificación de varios nuevos polimorfismos de nucleótido único (SNP) y otros genes asociados implicados en la patogénesis(1).

La exploración sistemática del paciente constituye un proceso esencial en el mantenimiento de la calidad de vida y evaluación del desarrollo de la enfermedad, por cuanto evaluación de hemograma, VSG, PCR, bioquímica general completa, sistemático de orina, estudio de coagulación, marcadores para VHB y VHC, EKG, radiografía de tórax, exploraciones

oftalmológicas periódicas de control y en pacientes femeninos, ginecológica constituyen algunos de los elementos fundamentales a evaluar, junto a electroforesis de proteínas $(11,25)$.

\section{Tratamiento.}

El tratamiento se prescribe en función de la gravedad de las manifestaciones, por lo general, el uso de colchicina, corticoterapia a nivel tópico o sistémica, inmunosupresión de ser necesaria y para formas más graves, y bioterapia en las formas refractarias(4).

\begin{tabular}{ll}
\hline Manifestaciones & Tratamiento \\
\hline Cutáneas & Colchicina, metotrexato , la Dapsona (pacientes in G-6PDH ), \\
glucocorticoides, Talidomida & Colchicina, la Sulfasalazina, dosis bajas de Corticoides, el Interferon- $\alpha-2-$ \\
Manifestaciones Articulares & a, Salazopirina 2-3 gr/día, prednisona $20 \mathrm{mg} /$ día, azatioprina 2,5 mg/kg/día, \\
& metotrexato 7,5-15 mg/semana, 3 veces a la semana, infliximab, etanercept \\
& y adalimumab. AINE (indometacina) \\
Aftas Orales Y Genitales & Tramiento tópico con acetonido de triamcinolona o betametasona, \\
& tetraciclina en enjuagues orales, sucralfato en enjuagues.Clorhexidina, \\
& Difenhidramina, o Hidrocortisona, solución de Lidocaína, corticoides \\
& tópicos o intralesionales, Sucralfato en suspensión, Pentoxifilina a 400 \\
& mg/8h \\
\hline
\end{tabular}




\begin{tabular}{ll}
\hline Neurológicas & Pulsos de prednisolona $1 \mathrm{gr} /$ día 3 días, seguidos de prednisona oral y \\
& ciclofosfamida $2,5 \mathrm{mg} / \mathrm{kg} /$ día o en bolos iv, infliximab, etanercept, \\
& adalimumab, clorambucilo $0,1-0,2 \mathrm{mg} / \mathrm{kg} /$ día, metotrexato. \\
& midriáticos y corticoesteroides locales, corticoides sistémicos, fármacos \\
Oculares & citotóxicos(Azatioprina $2,5 \mathrm{mg} / \mathrm{kg} /$ día y/o ciclosporina A $5 \mathrm{mg} / \mathrm{kg} / \mathrm{día}$,) \\
& infliximab, etanercept, adalimumab, clorambucilo 0,1-0,2 $\mathrm{mg} / \mathrm{kg} / \mathrm{dí}$, \\
& ciclofosfamida oral o iv, -interferón, tacrolimus. \\
Trombosis Venosa Profunda & Anticoagulación, corticoides, azatioprina, ciclofosfamida \\
Gastrointestinal & Prednisona 30-60 mg/día, salazopirina 3-6 mg/día, infliximab, etanercept, \\
& adalimumab, talidomida, cirugía. \\
Arterial & Prednisona 1-1,5 mg/kg/día con ciclofosfamida 2,5 mg/día oral o en pulsos \\
& mensuales de $1 \mathrm{gr}$, anticoagulación, técnicas endovasculares, cirugía.
\end{tabular}

Tabla 1. Tratamiento y dosificación en función de las manifestaciones clínicas (26-28).

\section{Conclusiones.}

- La Etiología del Síndrome de Behcet no está esclarecida, sin embargo, se puede aseverar que el componente genético está fuertemente relacionado, pero necesita de la intervención de un agente ambiental.

- Las manifestaciones que se presentan con más frecuencia son las implicaciones mucocutáneas, mismas que constituyen un factor fundamental en la prueba de patergia. Al evolucionar la patología, las manifestaciones a nivel ocular y vascular presentan mayor incidencia, mientras que el Neurobehcet, las manifestaciones articulares, gastrointestinales no suelen presentarse con frecuencia. El tratamiento de SB es proporcional al tipo y severidad de las manifestaciones clínicas, por cuanto es relevante conocer las opciones farmacológicas.

\section{Bibliográfia.}

- Muzeyyen Gonul, Selda Pelin Kartal, editores. Behcet's Disease [Internet]. InTech; 2017 [citado 15 de noviembre de 2017]. Disponible en:

https://www.intechopen.com/books/behcet-s-disease

- Yoshiaki Ishigatsubo, editor. Behçet's Disease [Internet]. Tokyo: Springer Japan; 2015 [citado 15 de noviembre de 2017]. Disponible en: http://link.springer.com/10.1007/978-4-431-54487-6

- Lorenzo Emmi, Domenico Prisco, editores. Behçet's Syndrome [Internet]. Milano: Springer Milan; 2014 [citado 15 de noviembre de 2017]. (Rare Diseases of the Immune System). Disponible en: http://link.springer.com/10.1007/978-88-4705477-6 
- Hié M, Amoura Z. Enfermedad de Behçet. EMC - Apar Locomot. septiembre de 2017;50(3):1-9.

- Castillo W, Gónzalez-Argote J, Hernández J. Enfermedad de Behçet. Rev Cuba Reumatol. 2014;16(3):309-21.

- Alfonso Valdés ME. Síndrome de Behcet. Rev Cuba Hematol Inmunol Hemoter. 2016;32(3):301-315.

- Solis Cartas U, Barbón Pérez OG, Bascó EL, Muñoz Balbín M, Molinero Rodríguez C, Solis Cartas E. Epidemiological clinical behavior of Behçet's disease. Rev Colomb Reumatol Engl Ed. abril de 2016;23(2):79-84.

- Michel Sánchez P, Valenzuela Ahumada F. Enfermedad de Behçet. Piel. junio de 2015;30(6):358-64.

- Singal A, Chhabra N, Pandhi D, Rohatgi J. Behcet's disease in India: A dermatological perspective. Indian J Dermatol Venereol Leprol. 2013;79(2):199.

- Maldini C, LaValley MP, Cheminant M, de Menthon M, Mahr A. Relationships of HLA-B51 or B5 genotype with Behçet's disease clinical characteristics: systematic review and meta-analyses of observational studies. Rheumatology. mayo de 2012;51(5):887-900.

- F Davatchi, Abdollahi Sadghei, F Sharam, A Nadji, C Chams-Davatchi, T Faezi, et al. Which Criteria to use for the Diagnosis of Behcet's Disease: International Study Group (ISG) Criteria or International Criteria for Behcet's Disease (ICBD)? J Immune Res. 2016;3(1).

- Molina RA, Huerta-Rosario A, Alva Díaz CA, Mejía Rojas KK, Mori N, Romero Sánchez R. Neuro-Behçet's disease in Peru: a case report and literature review. Medwave. 29 de junio de 2017;17(05):e6978-e6978.

- Scherrer MAR, de Castro LPF, Rocha VB, Pacheco L. Dermatoscopia no teste cutâneo da patergia: série de casos de pacientes com suspeita de Doença de Behçet. Rev Bras Reumatol. noviembre de 2014;54(6):494-8.

- Mayer MM, Ernst G, blo Young P, Barreira JC. Manifestaciones oculares en la enfermedad de Behçet. Front En Med. 2016;11(2):43-55.

- Takeuchi M, Karasawa Y, Harimoto K, Tanaka A, Shibata M, Sato T, et al. Analysis of Th Cell-related Cytokine Production in Behçet Disease Patients with Uveitis Before and After Infliximab Treatment. Ocul Immunol Inflamm. febrero de 2017;25(1):52-61. 
- García-Rivero AA, Gonzalez-Argote J, Martínez Larrarte JP. Neurobehçet. Rev Cuba Reumatol. 2015;17(3):222-233.

- Kirbas D. Neuro-Psycho-Behçet or Neuropsychiatric Behçet Disease: A Modified Name for an Old Disease. Noro Psikiyatri Arsivi. 2 de octubre de 2017;54(3):195195.

- Cañarte-Alcívar J, Lucas-Parrales E, Guerrero-Madroñero M, Moreira-Vinces R. Anemia en el adulto mayor. Polo Conoc. 2018;3(7):162-71.

- Morales-Angulo C, Vergara Pastrana S, Obeso-Agüera S, Acle L, González-Gay MÁ. Manifestaciones otorrinolaringológicas en pacientes con enfermedad de Behçet. Acta Otorrinolaringológica Esp. enero de 2014;65(1):15-21.

- Leal Gómez E, Campos Arca S, Fernández Rial M, Veiga González M. Síndrome de Behçet como diagnóstico diferencial de las úlceras genitales. Clínica E Investig En Ginecol Obstet. julio de 2014;41(3):138-41.

- Rogers RS. Recurrent Aphthous Stomatitis in the Diagnosis of Behçet's Disease. En: Behçet's Disease [Internet]. Berlin, Heidelberg: Springer Berlin Heidelberg; 2001 [citado 28 de febrero de 2018]. p. 119-28. Disponible en: http://link.springer.com/10.1007/978-3-642-56455-0_18

- Vaillant L, Samimi M, Parent D. Aftas, aftosis, enfermedad de Behçet. EMC Dermatol. junio de 2016;50(2):1-14.

- Cañarte-Alcívar J. Prevalencia de Vaginitis y Vaginosis en el Sector Gracias a Dios y Maldonado del Cantón Jipijapa en el 2012 [Internet]. Investigación Clínica y Epidemiología. Manabí 2012. 2012. Disponible en: http://investigadoressaludmanabi.blogspot.com/2012/06/dr-jorge-canarte-alcivarprevalencia-de.html

- Conde G, Yarelis S, Acosta Torres JR, Viltres Lescaille D, Ambos Frutos I, Oleaga Vargas S, et al. Características clínicas de la enfermedad de Behçet en pediatría. Rev Cuba Reumatol. 1 de abril de 2017;19(1):27-34.

- Howland-Alvarez I, Cañarte-Alcívar J, Guerrero-Madroñero M. Gammapatías Monoclonales en Portoviejo ¿Un diagnóstico sesgado? En: Primera Convención Científica Internacional de la UTM 2017 [Internet]. 1. ${ }^{\mathrm{a}}$ ed. Manabí, Ecuador:

Ediciones UTM; 2017. p. 303. Disponible en: https://www.utm.edu.ec/ediciones_utm/index.php/component/content/article/24libros/708-cci-utm-2017-libro-de-resumenes?Itemid=101 
- Valencia O, Guadalupe C, Guajardo G, Aleida D. Enfermedad de Behçet: Reporte de 2 casos. Rev Mex Oftalmol. :137-45.

- Calvo J, Baixauli A, Campos C, Gonzalez-Cruz I. Enfermedad de Behçet. En: Enfermedades reumáticas: Actualización SVR [Internet]. [citado 28 de febrero de 2018]. Disponible en: http://www.svreumatologia.com/wpcontent/uploads/2008/04/Cap-18-Enfermedad-de-Behcet.pdf

- Mayo Clinic. Diagnóstico y tratamiento [Internet]. 2 [citado 28 de febrero de 2018]. Disponible en: https://www.mayoclinic.org/es-es/diseases-conditions/behcetsdisease/diagnosis-treatment/drc-20351331? $\mathrm{p}=1$

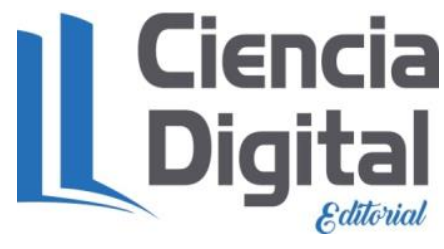


Para citar el artículo indexado.

Mera R., Cañarte J., Anzules J., Moreira R., Soasty R., Loor K.. (2018). Síndrome de Behcet, Revisión de la Literatura. . Revista electrónica Ciencia Digital 2(3), 616-632. Recuperado desde:

http://cienciadigital.org/revistacienciadigital2/index.php/CienciaDigital/article/view/179/15 $\underline{9}$

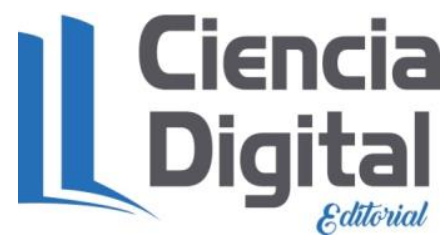

El artículo que se publica es de exclusiva responsabilidad de los autores y no necesariamente reflejan el pensamiento de la Revista Ciencia Digital.

El articulo queda en propiedad de la revista y, por tanto, su publicación parcial y/o total en otro medio tiene que ser autorizado por el director de la Revista Ciencia Digital.
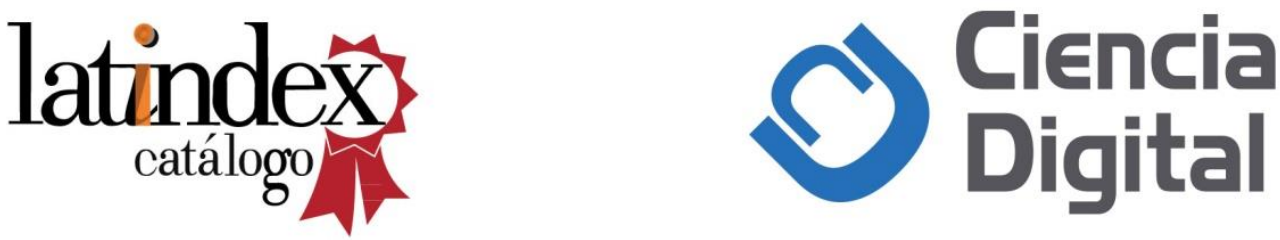